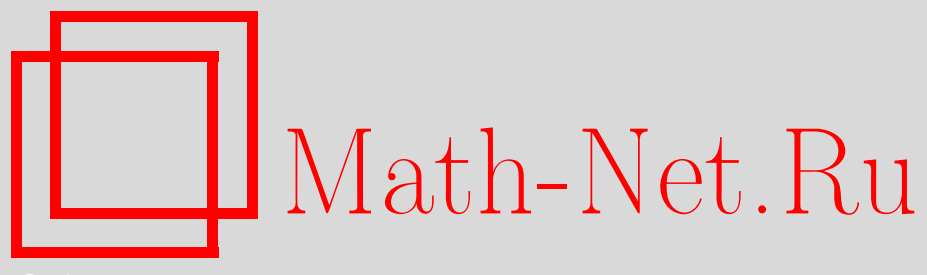

С. В. Асташкин, Новые экстраполяционные соотношения в шкале $L_{p}$-пространств, Функи. анализ и его прил., 2003, том 37, выпуск 3, 73-77

DOI: https://doi.org/10.4213/faa159

Использование Общероссийского математического портала MathNet.Ru подразумевает, что вы прочитали и согласны с пользовательским соглашением

http://www. mathnet.ru/rus/agreement

Параметры загрузки:

IP : 54.198 .55 .26

26 апреля 2023 г., 03:53:36

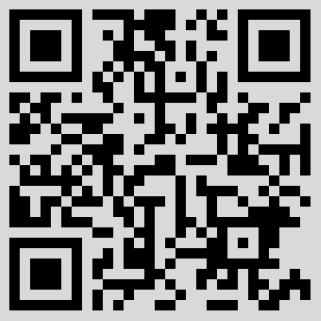




\title{
Новые экстраполяционные соотношения в шкале $L_{p}$-пространств
}

\author{
(c) 2003. С. В. АстАшкин
}

1. Введение. В 1951 г. Яно доказал, что всякий линейный оператор $T$, определенный на $\bigcup_{p>1} L_{p}[0,1]$ и при некотором $\alpha>0$ удовлетворяющий неравенствам

$$
\left(\int_{0}^{1}|T f(s)|^{p} d s\right)^{1 / p} \leqslant \frac{C}{(p-1)^{\alpha}}\left(\int_{0}^{1}|f(s)|^{p} d s\right)^{1 / p}
$$

для всех $p$ из какой-то правой полуокрестности точки $1(C>0$ не зависит от $p)$, является ограниченным оператором из пространства Зигмунда $L(\log L)^{\alpha}$ в пространство $L_{1}$ [1;2, c. 179-181]. Верно и двойственное утверждение: если линейный оператор $T$ ограничен в $L_{p}$ для всех достаточно больших $p$ и $\|T\|_{L_{p} \rightarrow L_{p}}=O\left(p^{\alpha}\right)(p \rightarrow \infty)$ при некотором $\alpha>0$, то $T$ является ограниченным оператором из $L_{\infty}$ в пространство $\operatorname{Exp} L^{1 / \alpha}$.

Позднее, в 90-е годы прошлого века, Яверс и Мильман предложили общие подходы к рассмотрению аналогичных ситуаций, заложив тем самым основы абстрактной теории экстраполяции операторов [3-5]. Основная цель этой теории заключается в изучении естественных предельных пространств, ассоциированных со шкалами пространств, а также оценок норм операторов, действующих в них. Библиографию по теории экстраполяции и ее многочисленным приложениям в анализе см. также в [3-5].

Используя сумму и пересечение семейств банаховых пространств в качестве конструкций (экстраполяционных функторов), приводящих к построению предельных пространств, Яверс и Мильман доказали ряд соотношений, из которых вытекают экстраполяционные теоремы об операторах, действующих в соответствующих пространствах. В частности, они получили следующую характеризацию экстраполяционных пространств, фигурирующих в теоремах Яно (см., например, [5, p. 22-23]):

$$
\Delta_{1<p<\infty}\left(p^{-\alpha} L_{p}\right)=\operatorname{Exp} L^{1 / \alpha} \quad(\alpha>0)
$$

и

$$
\Sigma_{1<p<\infty}\left((p-1)^{-\alpha} L_{p}\right)=L(\log L)^{\alpha} \quad(\alpha>0) .
$$

Основная цель данной заметки состоит в том, чтобы показать возможность восстановления, по крайней мере в случае шкалы $L_{p}$-пространств, не только отдельных предельных пространств, но и соответствующих их естественным парам интерполяционных функционалов. Благодаря этому удается ввести класс экстраполяционных функторов, равномощный вещественному методу интерполяции. Одновременно получены соотношения, более общие, чем (1) и (2), а также дано новое описание симметричных пространств, «близких» к пространствам $L_{\infty}$ и $L_{1}$.

В дальнейшем мы ограничимся рассмотрением пространств функций, определенных на $[0,1]$. Обобщения и усиления теорем Яно в случае функций, заданных на пространстве с $\sigma$-конечной мерой, см. в работах $[6,7]$. 
2. Экспоненциальные пространства Орлича и шкала $L_{p}$-пространств. Банахово пространство $X$ измеримых функций, определенных на $[0,1]$, называется симметричным, если из того, что $g^{*}(t) \leqslant f^{*}(t)$ и $f(t) \in X$, следует, что $g(t) \in X$ и $\|g\|_{X} \leqslant\|f\|_{X}$. Здесь и далее $f^{*}(t)$ - невозрастающая перестановка функции $|f(t)|[8$, c. 83$]$.

Для любого симметричного пространства $X$ имеют место непрерывные вложения $L_{\infty} \subset X \subset L_{1}$, где, как обычно, норма в $L_{\infty}-$ это $\|f\|_{\infty}=\operatorname{ess}_{\sup } \in[0,1]|f(s)|$ [8, c. 124-126].

Важный пример симметричных пространств - пространства Орлича. Если $N(t)$ - функция Орлича, т. е. возрастающая выпуклая функция на $[0, \infty), N(0)$ $=0$, то норма в пространстве Орлича $L_{N}$ определяется следующим образом:

$$
\|f\|_{L_{N}}=\inf \left\{u>0: \int_{0}^{1} N\left(\frac{|f(t)|}{u}\right) d t \leqslant 1\right\} .
$$

В случае $N(t)=t^{p}(1 \leqslant p<\infty)$ мы получаем обычные $L_{p}$-пространства, $\|f\|_{p}=\left(\int_{0}^{1}|f(t)|^{p} d t\right)^{1 / p}$. В дальнейшем нас будут интересовать экспоненциальные пространства Орлича $\operatorname{Exp} L^{\Phi}=L_{N_{\Phi}}$, где $N_{\Phi}(u)=e^{\Phi(u)}-1$, а $\Phi(u)-$ функция Орлича. Как известно $[9,10]$, норма в $\operatorname{Exp} L^{\Phi}$ эквивалентна норме в пространстве Марцинкевича $M(\varphi)$,

$$
\|f\|_{M(\varphi)}=\sup _{0<t \leqslant 1} \frac{\int_{0}^{t} f^{*}(s) d s}{\varphi(t)},
$$

построенном по вогнутой функции $\varphi(u)=u \Phi^{-1}(\ln (1+1 / u))$. Отсюда, в частности, следует, что $\operatorname{Exp} L^{\Phi}=\Lambda(\varphi)^{*}[8$, c. 152-154], где $\Lambda(\varphi)-$ пространство Лоренца, $\|f\|_{\Lambda(\varphi)}=\int_{0}^{1} f^{*}(s) d \varphi(s)$. Для $\Phi(u)=u^{\alpha}(\alpha \geqslant 1)$ получаем хорошо известные пространства Зигмунда $\operatorname{Exp} L^{\alpha}$ и $L(\log L)^{1 / \alpha}$ соответственно [11].

Пусть $X_{k}(k=1,2, \ldots)$ - банаховы пространства, линейно и непрерывно вложенные в линейное топологическое пространство $\mathscr{T}$. Тогда их пересечением называют банахово пространство $\Delta_{k=1}^{\infty} X_{k}$, состоящее из всех $x \in \bigcap_{k=1}^{\infty} X_{k}$, для которых $\|x\|=\sup _{k=1,2, \ldots}\|x\|_{X_{k}}<\infty$. Предположим дополнительно, что существует банахово пространство $X_{0}$, вложенное в $\mathscr{T}$, со следующим свойством: $X_{k} \subset X_{0}(k=1,2, \ldots)$ с константой 1 . Тогда сумма $\sum_{k=1}^{\infty} X_{k}$ определяется как множество всех $x \in X_{0}$, представимых в виде $x=\sum_{k=1}^{\infty} x_{k}\left(x_{k} \in X_{k}\right)$, где $\sum_{k=1}^{\infty}\left\|x_{k}\right\|_{X_{k}}<\infty$. Это пространство становится банаховым, если ввести на нем норму $\|x\|=\inf \sum_{k=1}^{\infty}\left\|x_{k}\right\|_{X_{k}}$, где нижняя грань берется по всевозможным представлениям $x$. И наконец, если $X-$ банахово пространство и $b>0$, то $b X-$ это пространство $X$ с нормой $\|x\|_{b X}=b\|x\|_{X}$.

Теорема 1. Для произвольной функции Орлича Ф справедливы следующие равенства (с эквивалентностью норм):

$$
\begin{aligned}
\Delta_{j=1}^{\infty}\left(\frac{1}{\Phi^{-1}\left(2^{j}\right)} L_{2^{j}}\right) & =\operatorname{Exp} L^{\Phi}, \\
\Sigma_{j=1}^{\infty}\left(\Phi^{-1}\left(2^{j}\right) L_{1+2^{-j}}\right) & =\Lambda(\varphi) .
\end{aligned}
$$


В случае $\Phi(u)=u^{1 / \alpha}(0<\alpha \leqslant 1)$ равенства $(3)$ и (4) переходят в дискретные аналоги равенств (1) и (2) соответственно.

3. Выражение интерполяционных функционалов пар $\left(L_{\infty}, \operatorname{Exp} L^{\Phi}\right)$ и $\left(\boldsymbol{L}_{\mathbf{1}}, \boldsymbol{\Lambda}(\boldsymbol{\varphi})\right)$ через $\boldsymbol{L}_{\boldsymbol{p}}$-нормы. В теории интерполяции операторов $[8,12,13]$ важную роль играют $\mathscr{K}$ - и $\mathscr{J}$-функционалы Петре, которые для произвольной пары $\left(X_{0}, X_{1}\right)$ и $t>0$ определяются следующим образом:

$$
\begin{aligned}
\mathscr{K}\left(t, x ; X_{0}, X_{1}\right) & =\inf \left\{\left\|x_{0}\right\|_{X_{0}}+t\left\|x_{1}\right\|_{X_{1}}: x=x_{0}+x_{1}, x_{0} \in X_{0}, x_{1} \in X_{1}\right\}, \\
\mathscr{J}\left(t, x ; X_{0}, X_{1}\right) & =\max \left\{\|x\|_{X_{0}}, t\|x\|_{X_{1}}\right\} .
\end{aligned}
$$

Соотношения (3) и (4) показывают, что пространства $\operatorname{Exp} L^{\Phi}$ и $\Lambda(\varphi)$ являются предельными для шкалы $L_{p}$-пространств. То же можно сказать и о пространствах $L_{\infty}$ и $L_{1}$, так как

$$
\Delta_{j=1}^{\infty} L_{2^{j}}=L_{\infty} \quad \text { и } \quad \Sigma_{j=1}^{\infty} L_{1+2^{-j}}=L_{1} .
$$

Аналогичное экстраполяционное описание имеет место и для интерполяционных функционалов пар $\left(L_{\infty}, \operatorname{Exp} L^{\Phi}\right)$ и $\left(L_{1}, \Lambda(\varphi)\right)$ (символ $\asymp$ будет означать существование двусторонних неравенств с константами, зависящими только от функции Орлича $\Phi)$.

Теорема 2. Если $\Phi-$ произвольная функцияя Орлича, то для любьх $f \in$ $\operatorname{Exp} L^{\Phi} u k=1,2, \ldots$

$$
\mathscr{K}\left(2^{k}, f ; L_{\infty}, \operatorname{Exp} L^{\Phi}\right) \asymp\|f\|_{\Delta_{j=k}^{\infty}\left(2^{k-j} L_{\Phi\left(2^{j}\right)}\right)}=\sup _{j \geqslant k}\left(2^{k-j}\|f\|_{\Phi\left(2^{j}\right)}\right) .
$$

Теорема 3. Если $\Phi-$ произвольная функциия Орлича, mо $\mathscr{J}\left(2^{-k}, g ; L_{1}, \Lambda(\varphi)\right)$ $\asymp\|g\|_{U_{k}}$, где $U_{k}=\Sigma_{j \geqslant k}\left(2^{j-k} L_{r_{j}}\right), r_{j}=1+\left[\Phi\left(2^{j}\right)\right]^{-1}$, для любьх $g \in \Lambda(\varphi) u$ $k=1,2, \ldots$.

Заметим, что ввиду вложений $L_{\infty} \subset \operatorname{Exp}^{\Phi}$ и $\Lambda(\varphi) \subset L_{1}$ с константой 1 имеют место равенства $\mathscr{K}\left(t, f ; L_{\infty}, \operatorname{Exp} L^{\Phi}\right)=t\|f\|_{\operatorname{Exp} L^{\Phi}}(0<t \leqslant 1)$ и $\mathscr{J}\left(t, g ; L_{1}, \Lambda(\varphi)\right)$ $=t\|g\|_{\Lambda(\varphi)}(t \geqslant 1)$.

4. Экстраполяционные функторы на шкале $L_{p}$-пространств и вещественный метод интерполяции. Если $E$ - банахова решетка двусторонних последовательностей вещественных чисел, то решетка $E\left(2^{-k}\right)$ состоит из всех $a=$ $\left(a_{k}\right)_{k=-\infty}^{\infty}$, таких, что $\left(a_{k} 2^{-k}\right)_{k=-\infty}^{\infty} \in E$ и $\|a\|_{E\left(2^{-k}\right)}=\left\|\left(a_{k} 2^{-k}\right)\right\|_{E}$.

Далее, $\Delta\left(\vec{l}_{\infty}\right)=l_{\infty} \cap l_{\infty}\left(2^{-k}\right), \Sigma\left(\vec{l}_{\infty}\right)=l_{\infty}+l_{\infty}\left(2^{-k}\right), \Delta\left(\overrightarrow{l_{1}}\right)=l_{1} \cap l_{1}\left(2^{-k}\right)$, $\Sigma\left(\overrightarrow{l_{1}}\right)=l_{1}+l_{1}\left(2^{-k}\right)$ и $e^{k}=\left(e_{j}^{k}\right), e_{k}^{k}=1, e_{j}^{k}=0(j \neq k), k=0, \pm 1, \pm 2, \ldots$

ОПрЕДЕЛЕНИЕ 1. Пусть $\Phi-$ функция Орлича, а $F-$ банахова решетка двусторонних последовательностей, $\Delta\left(\vec{l}_{\infty}\right) \subset F \subset \Sigma\left(\vec{l}_{\infty}\right)$. Тогда множество $\mathscr{L}_{\Phi, F}^{\mathscr{K}}$ состоит из всех измеримых на $[0,1]$ функций $f$, для которых последовательность $a_{f}=\Sigma_{k=0}^{\infty}\|f\|_{\Phi\left(2^{k}\right)} e^{k}$ принадлежит $F$.

Норма $\|f\|_{\mathscr{L}_{\Phi, F}^{\mathscr{K}}}=\left\|a_{f}\right\|_{F}$ превращает $\mathscr{L}_{\Phi, F}^{\mathscr{K}}$ в симметричное пространство. Так как ввиду (3) и первого равенства из (5) $\mathscr{L}_{\Phi, l_{\infty}}^{\mathscr{K}}=L_{\infty}$ и $\mathscr{L}_{\Phi, l_{\infty}\left(2^{-k}\right)}^{\mathscr{K}}=\operatorname{Exp} L^{\Phi}$, то $L_{\infty} \subset \mathscr{L}_{\Phi, F}^{\mathscr{K}} \subset \operatorname{Exp} L^{\Phi}$. 
ОПРедЕЛЕНИЕ 2 . Пусть $\Phi-$ функция Орлича, а $G$ - банахова решетка двусторонних последовательностей, $\Delta\left(\overrightarrow{l_{1}}\right) \subset G \subset \Sigma\left(\overrightarrow{l_{1}}\right)$. Обозначим через $\mathscr{L}_{\Phi, G}^{\mathscr{g}}$ множество всех измеримых на $[0,1]$ функций $g$, таких, что существует представление $g=\sum_{k=1}^{\infty} g_{k}$ (сходимость в $\left.L_{1}\right), g_{k} \in L_{r_{k}}, r_{k}=1+\left[\Phi\left(2^{k}\right)\right]^{-1}$, такое, что $\sum_{k=1}^{\infty}\left\|g_{k}\right\|_{r_{k}} e^{-k} \in G$.

Тогда $\mathscr{L}_{\Phi, G}^{\mathscr{J}}-$ симметричное пространство с нормой

$$
\|g\|_{\mathscr{L}_{\Phi, G}^{\mathscr{J}}}=\inf \left\|\sum_{k=1}^{\infty}\right\| g_{k}\left\|_{r_{k}} e^{-k}\right\|_{G},
$$

где нижняя грань берется по всем представлениям функции $g$ указанного вида. Так как ввиду (4) и второго из равенств $(5) \mathscr{L}_{\Phi, l_{1}}^{\mathscr{I}}=L_{1}$ и $\mathscr{L}_{\Phi, l_{1}\left(2^{-k}\right)}^{\mathscr{J}}=\Lambda(\varphi)$, то $\Lambda(\varphi) \subset \mathscr{L}_{\Phi, G}^{\mathscr{J}} \subset L_{1}$

Введенные конструкции пространств $\mathscr{L}_{\Phi, F}^{\mathscr{K}}$ и $\mathscr{L}_{\Phi, G}^{\mathscr{J}}$ можно рассматривать как экстраполяционные функторы на шкале $L_{p}$-пространств [3-5], значения которых можно найти, рассматривая соответствующие функторы вещественного метода интерполяции. Пусть $E$ - банахова решетка двусторонних числовых последовательностей. Если $\left(X_{0}, X_{1}\right)$ - произвольная банахова пара, то пространство $\mathscr{K}$-метода $\left(X_{0}, X_{1}\right)_{E}^{\mathscr{K}}$ состоит из всех $x \in X_{0}+X_{1}$, для которых $\|x\|=$ $\left\|\left(\mathscr{K}\left(2^{j}, x ; X_{0}, X_{1}\right)\right)_{j}\right\|_{E}<\infty$. В пространство $\mathscr{J}$-метода $\left(X_{0}, X_{1}\right)_{E}^{\mathscr{J}}$ входят все $x \in X_{0}+X_{1}$, допускающие представление $x=\sum_{j=-\infty}^{\infty} u_{j}$ (сходимость в $X_{0}+X_{1}$ ), где $u_{j} \in X_{0} \cap X_{1}$. Норма в $\left(X_{0}, X_{1}\right)_{E}^{\mathscr{J}}$ полагается равной

$$
\inf _{\left\{u_{j}\right\}}\left\|\left(\mathscr{J}\left(2^{j}, u_{j} ; X_{0}, X_{1}\right)\right)_{j}\right\|_{E},
$$

где нижняя грань берется по всем последовательностям $\left\{u_{j}\right\}_{j=-\infty}^{\infty}$, для которых имеет место указанное представление.

Предположим, что $E \supset \Delta\left(\vec{l}_{\infty}\right)$ (соответственно $\left.\{0\} \neq E \subset \Sigma\left(\overrightarrow{l_{1}}\right)\right)$. Тогда отображение $\left(X_{0}, X_{1}\right) \mapsto\left(X_{0}, X_{1}\right)_{E}^{\mathscr{K}}$ (соответственно $\left.\left(X_{0}, X_{1}\right) \mapsto\left(X_{0}, X_{1}\right)_{E}^{\mathscr{J}}\right)$ определяет интерполяционный функтор [13]. В частности, отсюда следует, что пространство $\left(X_{0}, X_{1}\right)_{E}^{\mathscr{K}}$ интерполяционно относительно банаховой пары $\left(X_{0}, X_{1}\right)$ (т.е. любой линейный оператор, ограниченный в $X_{0}$ и $X_{1}$, ограничен также в $\left.\left(X_{0}, X_{1}\right)_{E}^{\mathscr{K}}\right)$ (аналогичное утверждение справедливо для $\mathscr{J}$-метода). Совокупность всех таких функторов называется вещественным $\mathscr{K}$ - (соответственно $\mathscr{J}$-)методом интерполяции. Важно отметить, что в случае $\mathscr{K}$ - (соответственно $\mathscr{J}$-)метода решетку $E$ можно всегда считать интерполяционной относительно пары $\vec{l}_{\infty}=\left(l_{\infty}, l_{\infty}\left(2^{-k}\right)\right)$ (соответственно $\left.\overrightarrow{l_{1}}=\left(l_{1}, l_{1}\left(2^{-k}\right)\right)\right)$ [13, Corollaries $3.3 .10,3.4 .6]$. Будем называть $E$ в таком случае параметром $\mathscr{K}$ - (соответственно $\mathscr{J}$-)метода интерполяции.

Теорема 4. Для произвольной функции Орлича $\Phi$ и любого параметра $\mathscr{K}$-метода $F \quad \mathscr{L}_{\Phi, F}^{\mathscr{K}}=\left(L_{\infty}, \operatorname{Exp} L^{\Phi}\right)_{F}^{\mathscr{K}}($ с эквивалентностью норм $)$.

Как уже говорилось во введении, $\operatorname{Exp} L^{\Phi}=M(\varphi)$, где $\varphi(u)=u \Phi^{-1}(\ln (1+1 / u))$. Поэтому интерполяция в паре $\left(L_{\infty}, \operatorname{Exp} L^{\Phi}\right)$ описывается $\mathscr{K}$-методом [14]. Это 
означает, что всякое пространство $X$, интерполяционное относительно нее, представимо в виде $X=\left(L_{\infty}, \operatorname{Exp} L^{\Phi}\right)_{F}^{\mathscr{K}}$ для некоторого параметра $\mathscr{K}$-метода $F$. Тем самым мы получаем

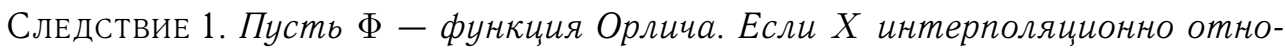
сительно пары $\left(L_{\infty}, \operatorname{Exp} L^{\Phi}\right)$, то существует параметр $\mathscr{K}$-метода $F$, такой, чmo $X=\mathscr{L}_{\Phi, F}^{\mathscr{K}}$.

Аналогичные результаты справедливы и в двойственной ситуации: для функтора $\mathscr{L}_{\Phi, G}^{\mathscr{J}}$ и пары $\left(L_{1}, \Lambda(\varphi)\right)$.

Теорема 5. Для произвольной функцзии Орлича $\Phi$ и любого параметра $\mathscr{J}$-метода $G \mathscr{L}_{\Phi, G}^{\mathscr{J}}=\left(L_{1}, \Lambda(\varphi)\right)_{G}^{\mathscr{J}}$ (с эквивалентностью норм $)$.

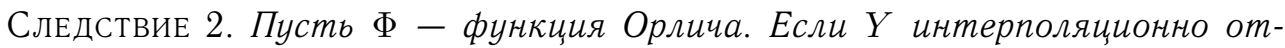
носительно пары $\left(L_{1}, \Lambda(\varphi)\right)$, то сущуествует параметр $\mathscr{J}$-метода $G$, такой, umo $Y=\mathscr{L}_{\Phi, G}^{\mathscr{J}} \cdot$

\section{ЛиТЕРАТУРА}

1. Yano S. J. Math. Soc. Japan, 3, 296-305 (1951). 2. Зигмунд A. Тригонометрические ряды. Т. 2. Мир, М., 1965. 3. Jawerth B., Milman M. Extrapolation Spaces with Applications. Mem. Amer. Math. Soc., 89, No. 44 (1991). 4. Jawerth B., Milman M. In: Israel Math. Conference Proc., Vol. 5, 81-105 (1992). 5. Milman M. Extrapolation and Optimal Decompositions with Applications to Analysis. Lect. Notes in Math., Vol. 1580, Springer-Verlag, 1994. 6. Carro M. J. J. Funct. Anal., 174, 155-166 (2000). 7. Carro M. J., Martin J. In: Function Spaces, Interpolation Theory and Related Topics. Proc. of the Intern. Conf. in honor of J. Peetre, Lund, Aug. 17-22, 2000, Walter de Gruyter, Berlin-New York, 2002, pp. 241-248. 8. Крейн С. Г., Петунин Ю. И., Семенов Е. М. Интерполяция линейных операторов. Наука, М., 1978. 9. Рутицкий Я. Б.УМН, 20, вып. 4, 205-208 (1965). 10. Lorentz G. G. Proc. Amer. Math. Soc., 12, 127-132 (1961). 11. Bennett C., Rudnick K. Diss. Math., 175, 5-67 (1980). 12. Берг Й., Лефстрем Й. Интерполяционные пространства. Введение. Мир, М., 1980. 13. Brudnyi Yu. A., Krugliak N. Ya. Interpolation Functors and Interpolation Spaces. North Holland Publish., 1991. 14. Cwikel M., Nilsson P. Math. Scand., 56. 29-42 (1985).

Самарский госуниверситет

e-mail: astashkn@ssu.samara.ru

Поступило в редакцию 23 октября 2002 г.

УДК 517.913

\section{Аналитическое доказательство неинтегрируемости АВC-течения при $\boldsymbol{A}=\boldsymbol{B}=\boldsymbol{C}$}

(c) 2003. С. Л. Зиглин

В работе продолжается исследование вопроса о существовании (непостоянного) первого интеграла у системы

$$
\dot{x}=A \sin z+C \cos y, \quad \dot{y}=B \sin x+A \cos z, \quad \dot{z}=C \sin y+B \cos x,
$$

